\title{
LA FILOSOFÍA DE LA CIENCIA Y LA FILOSOFÍA DE LA TÉCNICA EN RUSIA (1995-2000)
}

\author{
Ricardo BURGUETE AYALA \\ Ex Investigador Cientifico Superior \\ de la Academia de Ciencias de Rusia
}

Uno de los hechos que más sorprende a todo aquel que, en la situación actual de crisis económica, política e intelectual de Rusia, intenta profundizar en el estudio del proceso de evolución del pensamiento científico y filosófico en ese país, es, sin ningún género de dudas, el incremento durante los últimos años de las investigaciones dedicadas al estudio del propio pensamiento teórico y, en primer término, a la Filosofia de la Ciencia y la Filosofía de la Técnica. Un estudio, que va desde el origen y evolución del saber científico y el análisis de su estructura y características, hasta la vinculación socio-cultural y papel de la ciencia, la técnica y la tecnología en la historia de la civilización; pero siempre colocando en el centro de su investigación la importancia del progreso científico-tecnológico para el desarrollo futuro de la humanidad y su función en la situación actual de Rusia ${ }^{1}$.

1 Como ejemplo, solo citaremos los títulos de algunos de los libros que sobre estas cuestiones ha publicado los últimos cinco años el Instituto de Filosofia de la Academia de Ciencias de Rusia: Romanovskaya, T. La ciencia de los siglos XIX-XX en el contexto de la bistoria de la cultura. M., 1995. (142 pp.). Tr. colectivo, La filosofia de las partículas elementales. M., 1955. (217 pp.). Tr. colectivo: Los tipos históricos de racionalidad. T.I. M., 1995, (349 pp.) T.II. Moscú, 1996. (348 pp.). Tr. colectivo: La fisica en el sistema de la cultura. M., 1996. (231 pp.). OVCHINNIKOV, N.F. Los principios de teoretización del saber. M. 1996. (214 pp.). Tr. colectivo: La astronomía y el cuadro científico del mundo. M., 1996. (247 pp.). Tr. colectivo: Filosofia de la técnica: historia y actualidad. M., 1997. (283 pp.). Tr. colectivo: Ontologia y epistemología de la sinergética. M., 1997. (159 pp.). MaMCHuR E., OvCHINNIKov, N., OGurzov, A. La filosofia nacional de la ciencia: balance previo. Moscú, 1997. (359 pp.) YUdiN, B. La metodologia de la ciencia. Sistema, Actividad. M., 1997. PAVIENKO, A. La cosmología europea. Los fundamentos del cambio epistemologico. M., 1997. (156 pp.). RUSHAVIN, G. Los problemas metodologicos de la argumentación. M., 1997 (202 pp.) Tr. colectivo: La estrategia de la supervivencia. Cosmismo o 
Un hecho que sorprende positivamente, debido a que la historia y la filosofía de la Ciencia y la Técnica en Rusia inician su formación como disciplinas científicas solo en la segunda mitad del siglo XIX, y cuyo progreso se vio truncado, debido a que durante el largo periodo del poder Soviético el marxismo dogmático rechaza y combate estas disciplinas, que considera creaciones de la «ideología burguesa, ignorando las diferentes concepciones y teorías de la Ciencia y la Técnica que ya en los años 20-30 aparecen "Occidente». Sólo en los años 50, aún en los marcos ideológicos del marxismo, se empiezan a superar las limitaciones y prejuicios impuestos por el dogmatismo imperante y se crean las condiciones para un anális socio-cultural más profundo de la ciencia y el estudio del papel metodológico del saber científico, su estructura, niveles, métodos y su papel en el desarrollo de la cultura.

Desde entonces, estas disciplinas se fueron abriendo su propio camino en el sistema del conocimiento teórico, de tal modo que ya en los años 60-70 las investigaciones realizadas en este campo adquieren no solo cierta independencia, sino el amplio reconocimiento de su importancia y necesidad por la comunidad científica del país. El filósofo y científico ruso V. S. Stiopin describe la situación así: "En los años 60-70 la filosofía de la ciencia en nuestro país ya se considera una actividad prestigiosa para los filosofos. Fue, precisamente, en este campo, antes que en los demás, donde empezamos a superar el aislamiento ideológico y nos incorporamos a la discusión de los problemas sobre los cuales ya trabajaban nuestros colegas de Occidente. En este periodo, en nuestros trabajos sobre los problemas filosóficos de las ciencias naturales, los problemas ontológicos de la dialéctica de la naturaleza fueron pasando poco a poco a un segundo plano y un lugar muy importante ocupan las cuestiones de la lógica y la

Ideología. M. 1997, (3o4 pp.) KUZNEZOVA, N. Los problemas socio-culturales de la formación de ciencia en Rusia (siglo XVIII mediados del XIX). M., 1997. (264 pp.). Tr. colectivo: El contexto socio-cultural de la ciencia. M., 1998. (221 pp.) La técnica, la sociedad y el medio ambiente (Conferencia cientifica internacional. Moscú 18-19 de junio de 1998). M., 1998. (214 pp.). Tr. colectivo: La tecnología tradicional y la contemporánea. M., 1999, (216 pp.). ARSHINOV, V. La sinergética fenómeno de la ciencia postnoclásica. M., 1999. (203 pp.). SACHKOV YU. La revolución probabilistica en la ciencia. M., 1999. (143 pp.). Tr. colectivo: Las ciencias naturales en el contexto de humanidades. M., 1999 . (215 pp.). Tr. colectivo: El problema del status valorativo de la ciencia a comienzos del siglo XXI. Sant Petersburgo, 1999. (280 pp.). Tr. colectivo: Filosofia, Ciencia, Civilización. M., 1999. (367 pp.). STiopin, V. El saber teórico. M., 2000. (743 pp.). Tr. colectivo: Las fronteras de la Ciencia. M., 2000. (276 pp.). Tr. colectivo: La filosofia de las ciencias Naturales: mirada retrospectiva. M., 2000. (290 pp.). 
metodología de la investigación cientifica y, en primer término, los problemas de la estructura dinámica del saber cientifico" ${ }^{2}$.

Según este autor, fue precisamente en estos años cuando, tanto en la filosofía de la ciencia como en la historia de la filosofía y la lógica, aparecen «investigaciones originales y nuevas concepciones". Lo que se debe, entre otras cosas, a que el control ideológico sobre estas investigaciones no era ya tan riguroso como en las socio-políticas, pero también, al apoyo recibido por parte de la "comunidad científica no formal". Sin la ayuda de la cual no hubiéramos conseguido mantenernos ante la presión del totalitarismo" ${ }^{3}$.

La situación actual, que quizás pudiera parecer paradójica, se puede explicar de diferente modo. Se puede alegar, por ejemplo, como señala Stiopin, que a este tipo de investigaciones, ya desde los años 60 , se había incorporado un amplio grupo de especialistas, con una seria preparación profesional en la esfera de las ciencias naturales fundamentales (matemáticas, física, química, biología, etc.), que habían logrado conjugar estos conocimientos con un interés especial por la filosofía, la teoría y la historia de la ciencia. Se puede alegar también, que la alta profesionalidad de estos investigadores se convirtió en un punto de resistencia al dogmatismo simplista del marxismo oficial, adquiriendo en el sistema del saber oficialmente reconocido cierta independencia y personalidad propia. Fue, precisamente, desde las "filas de este colectivo" desde donde se inicia la batalla en defensa de la genética, la cibernética y las nuevas ideas en la física, en una palabra, contra todos aquellos seudocientíficos que, en nombre del marxismo dogmático, intentaron frenar el desarrollo de la ciencia en el país.

Evidentemente, todo esto ha contribuido decisivamente al desarrollo actual de estas investigaciones y su prestigio intelectual en la sociedad, así como al interés que despiertan las múltiples y serias publicaciones de estos últimos años. No obstante, la causa de que en la situación actual estas investigaciones no solo sean interesantes de por sí, sino de gran actualidad y resonancia social, radica en la importancia y el papel que la ciencia y la tecnología cumplen o están llamadas a cumplir en la etapa actual del desarrollo de nuestra civilización y en particular en los destinos de Rusia.

2 Stiopin, V. S. Entrevista con el Académico V. S. Stiopin en: Filosofia, Ciencia, Civilización. Moscú, 1999, pp. 355-356.

3 Ibid. p. 353. 
Por lo tanto, parece evidente, que una de las tareas más urgentes que la nueva situación plantea para los científicos y la ciencia en este país está directamente vinculada al cumplimiento de este papel que V. Stiopin define así: "Crear las premisas reales para el desarrollo postindustrial y resolver, por fin, una tarea ya planteada por la historia. El desarrollo postindustrial presupone no solo el mercado de mercancias y servicios, sino además de ideas y proyectos... Está claro por lo tanto, que es necesario invertir en la ciencia y no debemos destruir nada de aquellas estructuras organizativas que ya han demostrado su vitalidad" 4 .

La importancia de la ciencia en general en el cumplimiento de esta tarea y, en particular de la ciencia fundamental la señaló con cierta claridad el Presidente del País Vladimir Putin al intervenir en la reunión anual de la Academia de Ciencias el 22 de mayo del 2000 donde subrayó, que esta institución constituía una de las reservas estratégicas más importantes del país, que necesitaba no sólo el apoyo económico del estado, sino una seria reforma. Sus palabras fueron estas: "Ahora no estoy preparado para decir, qué y cómo debemos actuar, pero la Academia debe ser más contemporánea, más emprendedora, sentir el tiempo y sus exigencias. El estado tiene, naturalmente que ser más atento y más serio respecto a su reserva estratégica más importante" "5.

¿Significan estas palabras un posible cambio radical en la desastrosa política que el estado había practicado durante los últimos años en este campo? el tiempo lo dirá. Como es bien conocido, durante la anterior presidencia de B. Yeltzin se llevaron a cabo algunas acciones llamadas, presuntamente, a detener la destrucción del potencial científico heredado de la antigua URSS y propiciada por las conocidas "reformas". Incluso se intentó reactivar el desarrollo de la ciencia y se firmaron una serie de importantes documentos que reconocían la importancia de un cambio radical en este campo, que se consideraba una condición necesaria del desarrollo socio-político de Rusia. Lamentablemente, ni las correctas "recetas", ni las "buenas" intenciones se han llevado a la práctica. No obstante, su valor como directivas programáticas y vinculantes para el poder político siguen vigentes. En ellas, entre otras cosas, se exigía: «...apoyar

4 Los Académicos sobre la Academia. En la Revista Cuestiones de la Historia de las Ciencias Naturales y la Técnica. M. N.¹, 2000. p. 136.

5 "Nesavisimaya Gaseta» (Periódico Independiente) N. ${ }^{\circ}$ 5. 24. 5. 2000. Por cierto ha sido la primera vez que un presidente de Rusia participaba en las Reuniones anuales de la Academia de Ciencias. 
a la ciencia como una tarea prioritaria del estado;... crear las condiciones para el desarrollo de las investigaciones cientificas fundamentales;... elevar el prestigio del trabajo cientifico y crear las condiciones dignas para la vida y el trabajo de los cientificos y especialistas...; integrar la educación y la ciencia, desarrollar el sistema de preparación de cuadros cientificos cualificados..." ${ }^{6}$.

En este sentido, no han perdido su actualidad muchos de los planteamientos de otro importante documento de la etapa anterior, la Doctrina del desarrollo de la ciencia en Rusia, que a pesar de sus cuatro años de existencia, ni se ha llevado a la práctica, ni ha conseguido detener el proceso de desintegración del potencial científico e intelectual del país, del cual hablaremos más adelante. No obstante, quisiéramos citar algunos párrafos de uno de sus apartados iniciales titulado La ciencia es el recurso más importante de la renovación de Rusia, que hablan por si solos:

"La ciencia rusa en su multisecular historia ha contribuido decisivamente al desarrollo del pais y la comunidad mundial. Rusia debe en gran medida su condición de gran potencia mundial a los logros de los cientificos nacionales.

En las condiciones actuales la utilización práctica de los conocimientos cientificos naturales, humanistas y cientifico-técnicos se convierten cada vez en mayor grado, en una fuente que garantiza la vida de la sociedad su salud fisica y espiritual.

El nivel de desarrollo de la ciencia condiciona en alto grado la efectividad de la actividad económica, la defensa, la cultura espiritual y politica de la población del pais, la defensa del individuo y la sociedad de la acción de los factores naturales y antropogénicos dañinos»" .

En este mismo documento, al hablar del desarrollo del potencial científico de Rusia se afirma rotundamente, que el estado considera este potencial un bien nacional, que determina el futuro del país, por lo que su desarrollo debe ser una tarea prioritaria de aquel.

Pero los años han pasado, y las buenas y correctas palabras solo se han convertido en un cúmulo de nuevas realidades virtuales. Tal es el caso de otro documento aprobado por el gobierno en 1998, que, a pesar de incluir en su

6 UKAS del Presidente de la Federación Rusa del 13. 06. 96. N. ${ }^{\circ} 884$ "Sobre el desarrollo de la Ciencia en Rusia».

7 Rev. Cienciología. N. 2 . Moscú. 1999. p. 182. 
título la fecha exacta de su ejecución, la Concepción de la reforma de la ciencia Rusa para los años 1998-2000, tampoco se ha llevado a la práctica, por lo que el fatídico proceso desintegrador continua.

En estas condiciones, los científicos rusos continúan una lucha desigual contra los fiascos de las mal llamadas «reformas». Lucha, en la que uno de los aspectos más importantes es la defensa del papel y la importancia del saber científico en la historia de la civilización, en el desarrollo socio-económico de la sociedad y su valor y significado en las condiciones actuales de la Federación Rusa.

Para tener una visión más o menos precisa de lo que ha ocurrido y está ocurriendo en este país en el campo de la ciencia, hay que tener presente que en Rusia, como en todos los países que durante un largo periodo no acaban por encontrar un camino adecuado para su desarrollo económico-social, la situación de la ciencia y las características de su evolución han dependido y aún dependen, en gran medida, de una serie de circunstancias directamente vinculadas al cumplimiento de ciertas tareas concretas y urgentes que surgen en este proceso. De aquí la constante incertidumbre y los cambios bruscos en la actitud de la sociedad y sus grupos dirigentes hacia la ciencia en general y sus diferentes disciplinas y modalidades. Un hecho, que explica los vaivenes y altibajos en su desarrollo y las profundas contradicciones, que tanto saltan a la vista en la evolución histórica de la ciencia en este país durante el último siglo. Una situación que, no obstante, no pudo impedir que, ya a fines del siglo XIX principios del XX, los éxitos de la ciencia rusa y sus aportes al sistema mundial del saber científico fueran ampliamente reconocidos. Este camino, lleno de obstáculos y a su vez de éxitos espectaculares, se mantiene a lo largo de todo este siglo. Durante el cual, el factor fundamental que ha contribuido decisivamente al desarrollo de la ciencia han sido las necesidades económicas del desarrollo del propio país. Testimonio de esta realidad es el hecho, de que en las etapas cruciales de la historia de Rusia la ciencia ha constituido uno de los factores más importantes en todos los procesos de modernización. Ha sido en estos casos, cuando el interés por la ciencia como un medio de racionalizar y optimizar las diferentes esferas de la realidad, desde las relaciones económicas hasta la esfera de la cultura, se ha mantenido viva, rompiendo las trabas oficiales, religiosas, políticas e ideológicas.

Durante los períodos de modernización la ciencia se convierte «de repente" en la «hija preferida», no sólo del poder económico, sino también del político y hasta de una sociedad, a veces dominada por ideologías muy lejanas a la 
ciencia; mientras que en situaciones de crisis, estancamiento y tránsito se transforma en una "Cenicienta", en una pedigüeña inoportuna, que no recibe ni la atención ni los recursos necesarios para su desarrollo normal.

Durante los largos años del poder soviético se recurre a la ciencia cuando el país inicia ese difícil camino de la industrialización, que se intenta llevar a cabo con métodos autoritarios, buscando repetir e incluso mejorar los resultados obtenidos en los países occidentales, pero recorriendo este camino sin tener en cuenta o ignorando la existencia de las leyes y las normas de la economía de mercado.

La industrialización y la modernización del sistema económico presuntamente "socialista» se caracterizó por una relación muy compleja del poder político y la ciencia. Una relación, que evoluciona desde esa actitud negativa hacia la "ciencia capitalista", que caracteriza los primeros años post-revolucionarios y el intento utópico de crear una "Ciencia proletaria" diferente, implícito en la ideología del famoso "Proletkul», hasta un acercamiento y hasta integración de la ciencia rusa en el proceso mundial del desarrollo del saber científico en las últimas etapas. Un difícil camino en el que los éxitos y los fracasos son bien conocidos hoy día.

La desintegración de la Unión Soviética, el surgimiento de la Federación Rusa como un estado independiente y las reformas de los años 90 , que pretendían una modernización radical del País, han planteado la posibilidad y la necesidad urgente no sólo de conservar el potencial científico acumulado con tanta dificultad, sino de convertir a la ciencia en uno de los instrumentos básicos, que harían posible la realización de las transformaciones radicales que necesitaba la sociedad. Pero esto, como ya hemos señalado, no ha ocurrido.

La severa crisis socio-económica y política que acompaña el nacimiento de la nueva Rusia impulsa, se ha llegado a decir "obliga", a los reformadores que inician este proceso, no sólo a excluir a la ciencia de la esfera de sus prioridades, sino a tomar una serie de decisiones y medidas erróneas que llegan a poner en peligro la simple subsistencia de las instituciones científicas existentes, el desarrollo de las investigaciones y el sistema de enseñanza a todos los niveles, incluida la preparación de cuadros científicos. Un peligro que se ha conseguido, por ahora y a duras penas sortear, contra el "viento y marea de las reformas", con el trabajo y el sacrificio de los propios científicos y trabajadores de la ciencia, en primer término. Se ha creado una situación muy compleja y paradójica, que la prensa rusa de estos días describe así: "La situación actual de 
La ciencia en Rusia es un notable fenómeno del periodo de tránsito. A pesar de una durísima crisis económica y financiera, de una drástica disminución de las asignaciones de fondos estatales para las investigaciones cientificas y el estado calamitoso de la mayoria de los cientificos del pais, el sistema de investigaciones fundamentales antes creado aún sigue existiendo. El pais participa activamente en las labores de la comunidad cientifica mundial, las ponencias de nuestros cientificos en las conferencias internacionales despiertan gran interés y tienen un amplio auditorio y el número de artículos de autores rusos en las revistas cientificas de mayor autoridad es cada vez más importante. Y todo esto se lleva acabo cuando la situación interna en el campo de la ciencia es tan compleja y antinatural que no permite su funcionamiento normal y genera grandes pérdidas, a veces irreparables» ${ }^{8}$.

Para desvelar el «secreto» de esta situación paradójica e impugnar una serie de tópicos y mitos sobre el estado real de la ciencia en Rusia durante los últimos años, se han llevado a cabo serias investigaciones sociológicas, que basadas en documentos estadísticos oficiales y apoyadas por detalladas encuestas, han permitido establecer cierta claridad, tanto en la descripción real de los acontecimientos y la magnitud del proceso de desintegración de las estructuras científicas, como en la existencia de un considerable potencial científico aún no reclamado. Un potencial, que, basado en las investigaciones científicas fundamentales y aplicadas, está aún en condiciones de cumplir un importante papel en el desarrollo presente y futuro de la Federación Rusa.

Antes de la desintegración de la Unión Soviética las investigaciones científicas en ese país se desarrollaban institucionalmente por diferentes organizaciones subvencionadas por el Estado. Entre estas, por su importancia y magnitud, se pueden destacar: las vinculadas al sistema estatal de producción económica y a la defensa, las que pertenecían al sistema de enseñanza superior y media profesional (Universidades e Institutos especializados) y La Academia de Ciencias con sus múltiples Institutos y laboratorios.

El efecto de las reformas económicas no fue el mismo en cada una de estas esferas. La caída mas drástica se da en la ciencia que se había desarrollado en las diferentes ramas de la industria, en las que, durante estos últimos años, las inversiones del estado disminuyen en más de 6 veces, y en las que cerca del $80 \%$ de la producción científica no encuentra demanda y donde, según el

8 Nesavisimaya Gaseta. N. ${ }^{\circ}$ 5. 24 de Mayo, del 2000. 
Centro de Coyuntura Económica del Gobierno de Rusia, el desgaste de los fondos básicos de la industria ha alcanzado más de $50 \%$. Una industria donde por ejemplo, entre los años 1991-1995, la producción de tornos, en la cual la URSS era uno de los líderes mundiales, disminuye en 4,3 veces y donde en la extracción de petróleo solo el $10 \%$ de la producción se lleva acabo en la actualidad con tecnologías modernas, por lo que más de la mitad de las reservas establecidas no pueden ser extraídas. Por lo que no nos debe sorprender, que en todas estas ramas el número de trabajadores de la ciencia (investigadores y auxiliares), que en 1990 era de 1.500 .300 , en el año 1996 sólo fuera de 671.000 . Un descenso que supera con creces la media nacional, que disminuye algo más de dos veces, desde 1.943 .400 en 1990 hasta 946.000 en 1997. Aquí, la crisis que sigue a la desintegración del sistema económico llamado Socialista hizo verdaderos estragos, pero no tanto en las investigaciones fundamentales como en la ciencia aplicada y la tecnología, en las que en 1997 el número total de investigadores no pasa ya de los $455.000^{10}$.

Por todo lo expuesto, que es solo un ejemplo, parece evidente, que si los ritmos descendentes en la evolución de la ciencia rusa y en el campo de las innovaciones tecnológicas a ella vinculadas, que han tenido lugar esta última década, se siguen manteniendo durante varios años, la ciencia rusa dejará de ser, un componente significativo en el sistema de la ciencia mundial. Un proceso de retroceso que no ha llegado aun a su punto crítico. En la Federación Rusa aun trabaja el $12 \%$ de los científicos de todo el mundo, y sus investigaciones mantienen su prioridad en 17 de las 100 más importantes mundialmente realizadas, mientras que en 22 se acercan a ese nivel.

A fines de los años 80, la Unión Soviética ocupaba el primer lugar, entre los 8 países industriales más desarrollados, por el número de investigadores por cada 10.000 trabajadores, más de 120. En la actualidad esta cifra no llega a los 60 , mientras que en Japón y en los Estados Unidos ha aumentado constantemente y hoy día, su número es de 80 y 70 respectivamente ${ }^{11}$.

9 Noticias Financieras. 1998, 27 de mayo.

10 VARSHAVSKY, L. E.. El número y la estructura de los cuadros cientificos del pais: un visión del futuro. Cienciología. N. ${ }^{\circ} 1.2000$, p. 36.

11 Los datos que estamos utilizando están tomados de La ciencia en Rusia en cifras 1997. Moscú, 1997. y OECD. Main Science and Tecnology Indicators. París 1998, N. ${ }^{\circ}$ 2. y del artículo El potencial cientifico-técnico de Rusia: estado actual y problemas fundamentales de su desarrollo de LenCHuK, E. y STREPETOVA, M. Investigadoras del Instituto de Investigaciones Económicas y 
El destino de estos científicos desplazados del proceso investigador es muy variado, muchos encuentran otro tipo de trabajo en el país y algunos se incorporan al sistema de empresas privadas, que poco a poco se ha ido creando, pero un número considerable ha preferido continuar su trabajo profesional en el extranjero. Según los cálculos de los expertos, cada año las pérdidas por la fuga de cerebros alcanza en Rusia la suma de 50-60 mil millones de dólares. Los países más importantes hacia los que se ha dirigido esta emigración en los años 1996 y 1997 son respectivamente: Alemania 64,3\% y 63\%, Israel 17,8\% y 19,8\% y los Estados Unidos 12,3\% y 11,3\%. Por ejemplo a Israel entre 1989-1995 llegaron 11 mil científicos de la ex URSS y Rusia entre los cuales el 52\% eran físicos, el $27 \%$ biólogos y el $12 \%$ químicos.

No obstante la grave crisis económico-social de la que estamos hablando y la destrucción del potencial científico del país conducen, en el plano sociológico y cultural, a consecuencias de mayor alcance y, en primer término, a un descenso drástico de lo que la UNESCO ha definido como el Indice del Desarrollo del potencial Humano, en el que se incluyen una serie de factores (económicos, ecológicos, científicos, culturales, vitales, etc. ) y en el cual el llamado Potencial Intelectual de cada país ocupa un lugar de primer orden. Un potencial que, según el Human Development Report de 1999, ha descendido en Rusia, durante los años 90 del lugar 51 al 71.

Como se sabe para establecer el Potencial intelectual - (P.I.) existen diferentes parámetros y métodos. Uno de los más simples, pero bastante ilustrativo y representativo es el que utiliza los siguientes parámetros:

$\left(\mathrm{e}_{1}\right)$-nivel de educación de la población adulta;

$\left(\mathrm{e}_{2}\right)$ - peso especifico de los estudiantes en el conjunto de la población, según la proporción (1:10.000);

$\left(\mathbf{e}_{3}\right)$-gastos en Educación (en \% al) P.N.B.;

$\left(\mathrm{s}_{1}\right)$-el peso específico (en \%) del personal ocupado en el campo de la ciencia en relación con todos los ocupados;

$\left(s_{2}\right)$ - los gastos para la ciencia en relación al P.N.B en \%.,

y utilizando las siguientes formulas:

Políticas Internacionales de la Academia de Ciencias de Rusia. En la Revista Cienciologia $N{ }^{\circ} 3$. Moscú, 1999. pp. 9 - 29. 
P. E. (Potencial Educacional) $=\mathrm{e}_{1}+\mathrm{e}_{2}+\mathrm{e}_{3}: 3$

P. C. (Potencial Cientifico) $=s_{1}+s_{2}: 2$

permite establecer el potencial intelectual de cada país, utilizado la siguiente fórmula: P. I. = P. E. + P. C. : 2

Siguiendo este método, los cálculos realizados sobre la evolución del (P. I.) de la ex URSS y Rusia y sus perspectivas a corto plazo ofrecen las siguientes cifras ${ }^{12}$ :

\section{URSS}

$\begin{array}{lllll} & 1960 & 1989 & 1997 & 2002 \\ \text { P. E. } & 0,27 . & 0,51 . & 0,59 . & 0,51 . \\ \text { P. C. } & 0,31 . & 0,96 . & 0,35 . & 0,23 . \\ \text { P. I. } & 0,29 . & 0,71 . & 0,47 . & 0,37 .\end{array}$

Como se puede observar uno de los factores que más han incidido negativamente en el drástico descenso del potencial intelectual está directamente vinculado al estado de la ciencia donde solo el $\left(\mathrm{s}_{2}\right)$ que en 1989 era de 4,72 desciende en 1997 al 1,3. De lo que es responsable en gran medida el proceso caótico de desindustrialización, sobre todo del complejo industrial militar (incluidas las investigaciones cósmicas), donde estaba concentrada la mayor producción consumidora de innovaciones científico-tecnológicas del País.

Unos resultados similares, pero en cierto sentido diferentes y reveladores por su contenido y tendencia, nos ofrecen los estudios realizados a base de tres encuestas realizadas los años 1994, 1996 y 1998 en varios Institutos de la Academia de Ciencias, en los que las investigaciones fundamentales juegan un papel de primer orden e internacionalmente se consideran instituciones investigadoras de «élite». Tales son los Institutos moscovitas de Física, Química y Biología ${ }^{13}$.

12 RUTKEVICH, M. N. y LeVASHOV, V. K (Instituto de investigaciones socio-políticas de la Academia de Ciencias de Rusia) Sobre el concepto de potencial intelectual y los procedimientos de su medición. Revista Cienciología N. ${ }^{\circ} 1$ Moscú, pp. 49 -65. 2000

13 Un extenso y detallado resumen de los resultados de estas encuestas se publican en un número especial del periódico Nesavisimaya Gaseta el artículo de la Doctora en ciencias sociológicas Elena MIRSKAYA titulado La ciencia académica rusa en el espejo de la sociología. (Las cifras que señalamos en el presente artículo provienen de esa fuente). 
De este interesante y bien documentado artículo, que acabamos de mencionar en esta cita, queremos destacar algunos datos que reflejan con cierta nitidez el estado general de la ciencia en Rusia y, en particular la situación en la Academia de Ciencias, según la opinión de los propios científicos encuestados.

En las tres encuestas realizadas el estado general de las investigaciones científicas fue valorado como extremadamente crítico por el $86 \%$ de los encuestados, que señalaron como causas principales de esta situación: a) una financiación deficiente, el 75\%; b) la crisis socio-económica, el 68\%; c) los errores de la política estatal respecto a la ciencia, el 53\%; d) la no existencia de una estrategia adecuada en la reorganización de la ciencia, el $47 \%$.

En lo que se refiere al contenido y la calidad de las investigaciones (comparándolas con el nivel mundial): el 77\% (1994), el 73\% (1996) y el 62\% (1998) consideraron que no eran inferiores al nivel medio. Pero en 1994 el $23 \%$ consideró que lo superaba, mientras que en 1998 sólo el $8 \%$. A la pregunta sobre las perspectivas de su trabajo futuro en el campo de las ciencias y su disposición a hacerlo, la mayoría de los encuestados expresó sus deseos de continuar en estas labores y manifestó su esperanza de un cambio radical en la política científica, el 36\% en 1996 y el 43\% en 1998. En este caso estamos ante un testimonio evidente de que el pesimismo y la resignación de los científicos no es tan profundo como se suele pensar y se subraya insistentemente en muchos medios de comunicación. Según la última encuesta entre el 80$90 \%$ de los entrevistados no piensan abandonar su labor y vocación investigadora en el campo de la ciencia, ni tienen la intención de cambiar su orientación profesional. Se trata de una fidelidad sorprendente, si tenemos presente que durante los últimos años la situación económica de los propios científicos ha empeorado drásticamente, debido a que el crecimiento de los precios ha superado en varias veces el "aumento" de los salarios académicos y que el sueldo de la mayoría de los científicos está varias veces por debajo del mínimo vital, ya que en 1998 su capacidad adquisitiva había descendido en 10 veces.

En el artículo que estamos resumiendo se publican algunas cifras que merece la pena mencionar y se refieren a los sueldos académicos mensuales después de su última «subida» en abril del año $2000^{14}$.

14 En los Institutos Académicos existe un amplia escala de sueldos nos limitaremos a los extremos, que sin recurrir a la nomenclatura oficial los definiremos como Colaborador Científico (C.C.) y Colaborador Científico Superior.(C.C.S.). 
C. C.-entre 550 y 620 rublos +250 (doctorado) $=870=31 \$$, aprox.. 5580. pts.

C.C.S. -880 rublos $+420($ doctorado $)=1200=47 \$$, aprox. 8460 . pts. ${ }^{15}$.

Se trata, naturalmente, de sueldos «normales», pero según los cálculos de académico V. Guinsburg, el sueldo máximo que pueden alcanzar los doctores, que él define como "la fuerza fundamental de la ciencia", no logra sobrepasar con todos los posibles pagos adicionales los 1600 rublos $(57 \$$ o 10.260 . pts. al mes) ${ }^{16}$. Según la encuesta, las exigencias salariales de los científicos, en este sentido, son muy modestas. Mas de la mitad estaría conforme con recibir un sueldo de dos a cinco veces superior. Solo un $10 \%$ consideró que debería aumentar por lo menos 10 veces, con lo que aun estaría muy por debajo del sueldo recibido por sus homólogos extranjeros.

Como anécdota ilustrativa entre este cúmulo de cifras, nos ha parecido de cierto interés citar unas frases pronunciadas en tono humorístico por el conocido filósofo ruso V. Y. Tolstij en una de las sesiones del prestigioso «Club Teórico" del Instituto de Filosofía, en la que se discutía el tema El potencial intelectual de Rusia. Frases, que sin recurrir a los números reflejan con gran exactitud la situación que estamos describiendo.

"Cuando viajo al extranjero y me veo obligado a decir cuanto gana aqui, en Rusia, un doctor en ciencias o un profesor Universitario siempre oigo como respuesta: ¿Y Ustedes siguen trabajando? Hasta hace poco no nos creian, ahora nos creen, pero no dejan de sorprenderse...

.. Durante los últimos años, en nuestro Instituto de Filosofia somos testigos de un "Boom" de actividad y publicaciones. Hasta se ha llegado a decir, en forma de anécdota: que si mañana se suspenden los salarios, los investigadores seguirán escribiendo y publicando sus obras inmortales. La cabeza del intelectual esta constituida de tal modo, que no está vinculada directamente con el bienestar material, a pesar de que en esto, naturalmente, es mejor ser rico y estar sano, que ser pobre y estar enfermo."

${ }^{15}$ Los cálculos aproximados se hacen según el curso del mes de septiembre del 2000. $1 . \$=$ 28 rublos $=180$ pesetas.

16 Revista "Poisk" (Indagación). 1999 N. ${ }^{\circ}$ 52, p. 13. 
Y hacía la siguiente y triste reflexión:

"Al parecer, los que tienen el poder se basan en esta interpretación del mecanismo y el secreto del trabajo intelectual y por eso lo pagan tan mal" ${ }^{17}$.

Esta delicada situación se refleja también en la respuesta a la pregunta ¿Qué dificulta la realización de su labor investigadora? El 79\% señaló los bajos sueldos, el 57\% las dificultades en el proceso de experimentación, el 53\% la imposibilidad de realizar investigaciones de alto nivel y el 23\%, el sentimiento de que los resultados de su trabajo no se consideraran necesarios para la sociedad.

Según la autora de artículo, las encuestas han puesto en evidencia, que entre los científicos de la Academia, el interés por los trabajos colectivos de investigación se sigue manteniendo, que la colaboración con las instituciones extranjeras en esta materia es beneficiosa para el país y no estimula ni provoca el deseo de emigrar.

El último apartado del artículo, que la autora titula Mitos y realidad de la ciencia rusa, plantea y aclara una serie de cuestiones que, en gran medida, han distorsionado la valoración de la situación y las perspectivas del desarrollo de la ciencia en Rusia. Continuando el tema de la remuneración económica en el campo de la ciencia y la actitud de los científicos ante su trabajo, las encuestas han puesto en evidencia la falsedad de las afirmaciones de que los científicos obtienen buenas ganancias complementarias debido a los «muchos" trabajos que llevan a cabo fuera del ámbito científico profesional, donde muchos de ellos trabajan simultáneamente en dos o tres lugares. "Nuestros datos, afirma la autora del articulo, testimonian algo muy distinto: por regla general el trabajo complementario se lleva a cabo por los científicos en la esfera de la propia ciencia y la remuneración que reciben por ello es infima ${ }^{18}$.

La mitad de los científicos no obtiene ninguna ganancia fuera de la ciencia y no la quieren tener, ya que no consideran posible desviar su atención de su trabajo fundamental; un $30 \%$ quisiera tener ingresos complementarios en otras esferas de la actividad laboral, pero no encuentran la posibilidad de hacerlo y solo el $20 \%$ consiguen algún trabajo fuera de la ciencia. Estos son, en su inmensa mayoría jóvenes científicos. Por cierto, que sobre la función y

17 La Palabra Libre. Crónica intelectual 1998 - 1999. Moscú, 2000. p. 63.

18 Nesavisimaya Gaseta. N. ${ }^{\circ}, 5.24 .05 .2000$. 
la situación de los jóvenes investigadores en la Academia de Ciencias se han formulado versiones muy diferentes y contradictorias.

Durante los años 80 principios de los 90 se divulgó con cierta insistencia la idea de que la situación de los jóvenes en las instituciones académicas era insoportable, que los viejos cuadros discriminaban y cerraban el camino a los jóvenes talentos, que la Academia envejecía, perdía su vigor e iniciativa, estaba al borde de la catástrofe generacional. Mas tarde este cuadro se intentó "subsanar" con declaraciones poco fundadas sobre los presuntos privilegios, facilidades y perspectivas halagüeñas, que esperaban a las nuevas generaciones de científicos que se incorporaban a los trabajos de investigación académica. Las encuestas han demostrado que ni lo uno ni lo otro correspondía a la realidad, que estas afirmaciones habían intentado generalizar tendencias y situaciones puntuales muy concretas. Esto significa, que ni la discriminación deliberada, ni la cacareada atención especial, se podían colocar en un primer plano a la hora de hacer una valoración global. Lo que no obstante, si se puede afirmar es, que en los marcos de la Academia no se ha llevado a cabo una utilización adecuada de los jóvenes científicos. En el artículo esta situación se resume así: "El sequimiento del año 1996 (se refiere a la encuesta) ha mostrado, que solo un número muy reducido de jóvenes participa en las investigaciones de élite. $Y$ esto implica, que las formas existentes de apoyo a la juventud no estimulan el crecimiento cientifico de las nuevas generaciones $y$, por lo tanto, no garantizan una sustitución en perspectiva. En la práctica de la ciencia mundial se ha establecido que la actividad profesional de los científicos en el campo de las ciencias naturales alcanza su máxima efectividad desde los 30 a los 40-45 años, mientras que en los colectivos investigativos de la Academia los mejores resultados los obtienen representantes de grupos de mayor edad, entre 41-50 y 51-60 años, lo que es completamente anormal para el funcionamiento estable de la ciencia y crea una situación de "rascar el fondo" del recipiente" 19.

Otra de las cuestiones, que ha despertado gran interés en los medios de comunicación y se discute ampliamente en la sociedad, es el tema de la emigración de los científicos o la llamada "fuga de cerebros", de la que ya hemos ofrecido algunos datos generales. Existe la opinión muy extendida y ampliamente reflejada en los medios de información, de que esta "fuga" se incrementa y sigue adquiriendo dimensiones catastróficas. Se ha llegado a decir, que 
casi todos los científicos que aún quedan en el país solo esperan la ocasión para abandonarlo, y que ante ellos se plantea el dilema: si se quedan en Rusia tendrán que abandonar la ciencia, si piensan dedicarse a ella deberán abandonar el país.

Otra vez, las encuestas no han confirmado ni la veracidad de la tendencia señalada, ni la existencia de tal dilema. Las de 1996 y 1998 muestran, que cerca de un $43 \%$ de los encuestados no quiere bajo ningún concepto abandonar el país; entre el $40-45 \%$ estarían dispuestos a trabajar en el extranjero, pero por un tiempo limitado y solo el $2-3 \%$ quisieran irse definitivamente. Es necesario destacar, que desde el año 1996 el movimiento migratorio de esta parte de la población se ha convertido en un viaje de ida y vuelta, en el que el número de potenciales emigrantes ha disminuido drásticamente del $20 \%$ al $2 \%$. Un viaje durante el cual muchos científicos se han dado cuenta de que para las instituciones científicas extranjeras el interés hacia ellos se debía, en primer término, al hecho de ser los representantes de ciertos colectivos investigadores que realizaban en su país serias e interesantes investigaciones.

No se puede negar que durante los años 90 el número de investigadores en los diferentes Institutos de la Academia de Ciencias se haya reducido, la crisis económica los ha afectado sensiblemente. Según los datos de la encuesta en un $18,6 \%$, es decir, aproximadamente en unas 12 mil personas. Una cifra que implica, no sólo un estancamiento, sino también cierto retroceso, pero no una catástrofe. En el plano cualitativo, después de una década muy compleja, la ciencia académica sigue conservando su potencial intelectual creativo. Tres factores han contribuido a ello: ha contado con cierto apoyo económico (mínimo e insuficiente) por parte del Estado y la Sociedad, ha mantenido intactas en lo esencial, sus estructuras organizativas y ha contado con la increíble fidelidad y paciencia de la comunidad científica, que no ha perdido la fe y la esperanza de que la crisis económica tocaría fondo y que sus servicios serían requeridos por la sociedad, que acabaría por comprender que su "reserva estratégica fundamental» sería, en última instancia, el instrumento que permitiría al país salir definitivamente a la superficie.

Por todo lo expuesto, parece comprensible que el análisis de la interrelación y el condicionamiento mutuo del desarrollo de la ciencia y la sociedad, así como el estudio de los múltiples problemas que la evolución de la sociedad y la cultura plantean al pensamiento teórico, sea en la actualidad uno de los temas centrales y prioritarios que la Filosofía de la Ciencia y la Filosofía de la Técnica abordan en sus investigaciones. También es evidente que, por su 
actualidad e importancia, estos temas exijan, a veces, respuestas concretas, rápidas y operativas. Las que, con mucha frecuencia, no han encontrado aun soluciones teóricas adecuadas. En estos casos, la práctica social no puede esperar las soluciones que se proponen en los trabajos académicos. Evidentemente, un lugar más adecuado para discutir las diferentes ideas son las "mesas redondas», simposios, conferencias y las páginas de las Revistas especializadas, posibilidades que en la Rusia actual se utilizan ampliamente ${ }^{20}$.

Otro de los graves e importantes problemas que durante estos últimos años ha preocupado tanto a la comunidad científica como a la sociedad en su conjunto, está vinculado a la "proliferación" en el país de ciertas doctrinas y presuntas teorías que, de una u otra forma, se intentan oponer a la ciencia. Unas para impugnar sus soluciones concretas y el alcance del saber científico en general, otras para suplantarla, o medrar a su sombra. Unas utilizando el hecho de que existen determinadas zonas de la realidad humana que no son abarcadas por la ciencia y el racionalismo científico y son reflejadas y expresadas por otras formas del conocimiento, cuyo valor socio-cultural no se puede negar, a pesar de su status no científico y otras, simplemente especulando con los prejuicios y la ignorancia de parte de la población, para divulgar ideas anticientíficas.

Esta realidad exige, no solo una rigurosa diferenciación del saber científico y no científico, sino en los marcos de este último, una nítida distinción de la no-ciencia de la anti-ciencia en los marcos de la cual se deben incluir la paraciencia y la seudociencia.

En esencia, ese conjunto de "falsas teorías", que definimos como anti-ciencia, se forma al intentar transferir al saber común o a la «sabiduría popular» ciertas ideas tomadas de la astrología, la magia o la experiencia religiosa, utilizando para ello la terminología o, mejor dicho, la «fraseología» científica. Lamentablemente este fenómeno no solo es característico de la Rusia actual. Aquí, en España también están de moda los pronósticos astrológicos, los magos adivinos, los extrasens y los prejuicios religiosos. No obstante, estas ideas, testimonio de una educación formativa deficiente, no representan un serio peligro para el desarrollo de la ciencia.

20 Además de las revistas especializados los resultados de estas discusiones se publican en los medios de comunicación y en ediciones especiales como por ejemplo el anuario La Palabra Libre y la nueva revista Cienciologia que se publica trimestralmente desde 1999. 
El verdadero peligro en la etapa actual proviene de la propia ciencia, y es dentro de esta de donde se pueden esperar serios contratiempos y dificultades, no solo en el proceso de su desarrollo teórico, sino en la interpretación y aplicación de sus resultados concretos. Este peligro ha existido siempre, hasta se puede decir que es una de las características del proceso interno del desarrollo de la ciencia, de su paso de un nivel a otro, de un tipo de racionalidad científica a otro. Es cierto, que con frecuencia las concepciones anticientíficas que surgen en el seno de la propia ciencia tienen un marcado carácter personal. Esto ocurre cuando determinados científicos por error o ambición no saben valorar el alcance real de sus investigaciones e intentan utilizar determinados resultados parciales para formular presuntas leyes y teorías "universales» e incluso para proponer un cuadro del mundo diferente.

Según V. S. Stiopin, al hablar de Rusia: «Se pueden destacar cuatro causas sociales que muestran boy dia la gravedad del problema de la relación de la ciencia y la seudociencia.

La primera es la crisis general de la civilización y la búsqueda de nuevos valores; la segunda, son las particularidades de la sicologia y la mentalidad del desarrollo postindustrial; la tercera, el estado de la propia ciencia, vinculado al retraso de los procesos de integración del saber cientifico, que cada dia se presenta más diferenciado, y por último a las características de la vida en La propia Rusia» ${ }^{21}$.

Lo mas preocupante de la situación actual, radica en que hoy, a las voces, ya desacreditadas de los críticos "tradicionales" del saber científico y la función de la ciencia como institución social, se añaden las de algunos que, por su vocación y dedicación profesional, se pueden considerar actores activos del propio proceso científico de investigación. Aquí se deben de incluir, desde los que critican a la ciencia con ideas supuestamente humanistas y ecologistas, hasta aquellos que desde diferentes concepciones teóricas ("constructivismo social», "sociología del conocimiento", "postmodernismo", etc.) intentan demostrar que la ciencia debe renunciar a cumplir un importante papel en la historia de la cultura, debe abandonar sus pretensiones de ser la portadora de un saber verdadero.

En las circunstancias de la Rusia de hoy, el peligro más serio proviene de ese movimiento cultural que se conoce con el nombre de Postmodernismo y

21 StIopin, V. S. La ciencia y la seudociencia. Revista Cienciología. N. ${ }^{\circ} 1$. Moscú, 2000. p. 77. 
que en la actualidad se desarrolla en líneas paralelas en la cultura (el arte, la literatura, la pintura, la música, etc.) y en la ciencia y la tecnología, contaponiendo en forma radical a los principios y leyes fundamentales de la ciencia clásica los resultados concretos de la ciencia post-no-clásica.

Ahora no queremos entrar en el análisis detallado de este complejo fenómeno, ni en los orígenes del término en si, ni tampoco trataremos sobre los diferentes contenidos, que tanto los partidarios como los críticos de las ideas del "Postmodernismo cientifico" le adjudican. No obstante, se debe señalar, que este "movimiento" surgido a principios de los años 60 , y que se presenta en su etapa inicial como una corriente estético-literaria, alcanza muy pronto la magnitud de una forma de pensamiento crítico-filosófico de la modernidad (Lyotard, Derrida, Foucault y muchos otros), y se ha convertido en la actualidad en un importante fenómeno de la conciencia social de la época postindustrial.

En la ciencia, las ideas postmodernistas penetran como una reacción crítica a la supuesta crisis del racionalismo de la Ciencia Clásica y su papel rector en el ámbito del pensamiento teórico. Una crítica que inculpa a la ciencia de la Epoca Moderna, y con ella a la ciencia en general, de ser una de las causas fundamentales de los males y desajustes que aquejan a la sociedad de nuestros días, de imponer a la cultura no solo un tipo de racionalismo y unos métodos del conocimiento inadecuados, sino un modelo socio-cultural absoleto. Una ciencia, que no solo se declara responsable de los hechos del pasado, sino que, presuntamente, sigue siendo un obstáculo que no permite superar los estrechos marcos de la "modernidad", "implicando un enorme peligro para la vida del hombre en el Planeta» 22.

En la actualidad, en las ideas postmodernistas sobre la ciencia existen varias tendencias, que se pueden distinguir por su actitud hacia la ciencia contemporánea. Una actitud que va desde la crítica más intransigente, hasta la búsqueda de cierto compromiso con la concepción y los principios que caracterizan la ciencia de nuestros días. Por ello, cuando asociamos los términos "postmodernismo» $y$ «anticiencia», nos referimos, fundamentalmente, a esa primera actitud o corriente, cuyo contenido se conoce como "Versión fuerte» y cuya

22 Grifin, D. R., Smith. H. Primordial Truth and Postmodern Therory. New York, 1989 pág. XIII. 
esencia E. A. Mamchur define así: «El postmodernismo critica el logro fundamental de la modernidad-la neutralidad de las ciencias naturales. A diferencia de la modernidad que se caracteriza por su fe en la ciencia y el progreso, en la primacía absoluta del pensamiento racional, el postmodernismo promueve la desconfianza en la ciencia y proclama su intención de substituirla por cierto saber alternativo» ${ }^{23}$.

A primera vista puede parecer que uno de los puntos fuertes en la argumentación "postmodernista» son sus constantes referencias a algunos de los planteamientos y principios formulados en la ciencia post-no-clásica ${ }^{24}$. Principios, que se intentan oponer en forma radical y excluyente al pensamiento científico precedente. Tal es el caso, para citar algunos ejemplos, del "relativismo absoluto», de la impugnación radical del principio de objetividad del saber científico y el realismo de las teorías científicas o la divulgación de la idea de la pluralidad de mundos posibles.

Con esta argumentación los partidarios de la versión fuerte intentan demostrar que la concepción de la ciencia que mantienen la mayoría de los científicos ya no refleja el verdadero estado de las cosas y no corresponde al nivel de desarrollo del saber científico contemporáneo. Por otra parte, argumentan también que, cuando examinamos la función que cumplen las ciencias en diferentes culturas y el papel que juegan en la realización de los diferentes proyectos sociales, no podemos hablar de una sola ciencia, sino de una pluralidad de diferentes tipos de concepciones científicas, pragmáticamente orientadas. Pluralidad que surge debido a que no existe una realidad objetiva, sino una pluralidad de mundos, que se crean durante el propio proceso cognoscitivo. "Lo más importante, - decía un postmodernista ruso-, es comprender de una vez, que de por si no existe ninguna naturaleza fuera de nuestra actividad intelectual y práctica" 25 .

En la situación actual de Rusia, la divulgación de esta posición extrema es, sin dudas, bastante preocupante, hasta se puede decir peligrosa. Esto se puso en evidencia con gran precisión, en la amplia polémica que caracterizó

23 MAMCHUR, E. A. ¿Se puede aplicar la concepción de los posibles mundos al mundo del saber científico. Rev. Cienciología. N. ${ }^{\circ}$ 2. Moscú, 1999. pp. $127-128$.

24 También intenta capitalizar y reinterpretar las ideas de conocidas figuras del campo de la historia la sociología y la filosofía de la Ciencia desde el relativismo cultural de Spengler hasta la filosofía social de K. Popper.

25 Periódico Independiente. N. ${ }^{\circ}$ 5. 24. 05. 2000. 
el discurso de los participantes de dos "Mesas redondas» que tuvieron lugar a principios de este año, con gran resonancia en los medios de información masiva y fueron publicadas en el Suplemento "Ciencia" del "Periódico Independiente» (Nesavisimaya Gaseta). La primera en el N. ${ }^{\circ} 2$ del 16 de febrero, cuya iniciariva de convocatoria y organización perteneció, precisamente a ese grupo de "científicos críticos", que formularon el tema de discusión en los siguientes términos: Las perspectivas de la racionalidad científica. ¿Se pondrá fin en el próximo siglo al dictado de las ciencias naturales?

La segunda, que, como réplica a la primera, organiza el Instituto de Filosofía de la Academia de Ciencias, tiene lugar el 29 de marzo y formula su tema central así: El status de la Ciencia en la Cultura contemporánea. Los materiales de este evento se publican parcialmente en el mismo periódico, el 24 de mayo ${ }^{26}$.

La discusión ha puesto en evidencia una vez más, no solo la importancia de estos temas, sino la existencia dentro de la propia comunidad científica de una gran diversidad de opiniones, puntos de vista y conclusiones teóricas sobre estas cuestiones, que intentan basar sus planteamientos en diferentes criterios sociológicos, filosóficos y científicos, pero como hemos intentado demostrar, también paracientíficos.

No sería justo afirmar que todas las cuestiones planteadas han sido resueltas, pero parece evidente, que algunas afirmaciones de los partidarios de la "Versión fuerte" han sido rechazadas con cierta rotundidad. Por ejemplo la afirmación de que "El saber cientifico se construye integramente por las circunstancias sociales y solo depende de ellas". o que "El mundo de la naturaleza como objeto de estudio y como condicionante del contenido del saber cientifico desaparece y no es necesario" ${ }^{27}$. Han sido rechazadas por todos aquellos que consideran que el Mundo que estudia la ciencia es Uno y Una es la verdad que esta refleja. Que en su desarrollo la ciencia es cada vez más exacta, y sus resultados son cada vez más adecuados a esa pretendida verdad. Que si esto es así, la pluralidad de concepciones y teorías que caracteriza cada etapa histórica en el desarrollo de la ciencia, incluida la actual, debe converger en una teoría única. Teoría, que no

26 El texto integro de las intervenciones en esta «Mesa Redonda» se publicará en el próximo N. ${ }^{\circ}$ del Anuario "La palabra Libre», en estos momentos en la Imprenta.

27 Markova, L. A. ¿Una ciencia, un mundo? Rev. Cienciologia. N. ${ }^{\circ} 1$. Moscú, 2000, p. 143. 
es una etapa final, sino un punto de partida, que inicia el camino hacia una nueva diversidad y una nueva síntesis.

La discusión ha dejado bien claro que la ciencia "no-clasica» y "post-no-clásica no niega la etapa anterior, ni los principios fundamentales que la inspiran, sino que intentan superar su absolutización por ciertas tendencias de origen filosófico que, a lo largo de todo el siglo XX, han querido imponer sus criterios a la ciencia. Entre estas el neopositivismo, el idealismo subjetivo y ahora la "versión fuerte" del postmodernismo.

Todo lo señalado, quizás pueda explicar porqué un hecho que, a primera vista pudiera haber parecido sorprendente y paradójico, tiene una motivación lógica y representa una reacción adecuada ante una situación histórica concreta. Situación, que condiciona, en cierta medida, las características, el contenido y el énfasis de este "boom» investigador en el campo de la filosofía y la historia de la ciencia y la tecnología.

Una mirada retrospectiva sobre la estructura sistemática de la abundante literatura publicada sobre estos temas permitirá, en rasgos generales, establecer una clasificación de estos, por su forma y contenido. La que según nuestra apreciación se puede agrupar en varias direcciones temáticas fundamentales.

a) El estudio de las caracteristicas y el significado del pensamiento teórico en general y el cientifico en particular. En esta dirección se pueden destacar monografías como El saber Teórico del Académico V. S. Stiopin, ${ }^{28}$ en la que como señala su autor "se exponen los resultados de más de veinte años de investigaciones de la estructura y la dinámica del saber teórico de la ciencia» (p. 8.) ${ }^{29} \mathrm{O}$ la mono-

28 Stiopin, V. S. Director del Instituto de Filosofía de la Academia de Ciencias de Rusia, Presidente de la «Sociedad filosófica» de Rusia. Autor de más de 250 trabajos científicos entre ellos las monografías: El positivismo contemporáneo y las ciencias particulares. MINSK, 1963; La naturaleza práctica del conocimiento y los problemas metodológicos de la fisica contemporánea. MINSK, 1970; Los métodos del conocimiento científico. MINSK, 1974; La formación de la teoria cientifica. MINSK, 1976; La naturaleza del conocimiento cientifico. MINSK, 1979; Los ideales y las normas del conocimiento científico. (Redactor y coautor). MiNSK, 1981; Las revoluciones cientificas en la dinámica de la cultura. (Redactor y coautor). MiNSK, 1987; Introducción a la Filosofia. T.T. 1 - 2 (Coautor). Moscú, 1989; La Antropologia filosófica y la historia de la ciencia. Moscú, 1992; La formación y las normas de la ciencia post-no-clásica. En el libro Problemas de la metodología de la ciencia post-no-clásica. Moscú 1992; La filosofia de la ciencia y la técnica. /Coautor); Moscú, 1995; El Saber Teórico. Moscú, 2000.

${ }^{29}$ Como las fichas bibliográficas de los trabajos que vamos a citar a continuación se ofrecen al inicio de este artículo, a continuación en el texto solo ofreceremos entre paréntesis el N. ${ }^{\circ}$ de las pp. citadas. 
grafía de N. F. Ovchinnikov Los Principios de Teoretización del Saber, en la que se analiza el proceso de construcción de las teorías científicas y los principios metodológicos que constituyen el fundamento del saber teórico, su génesis y desarrollo en el proceso de evolución del conocimiento científico. Un tema que el autor examina en el contexto general de la Cultura. "La ciencia, dice el autor, no es nada más que un elemento de un fenómeno integral, sólo es una parte del sistema integral de la actividad creadora del hombre, que se alza sabre el fundamento de ese fenómeno tan dificil de definir que llamamos cultura. Al analizar los principios del saber teórico de carácter cientifico y al describir su funcionamiento en la vida de las teorías cientificas no hemos podido abarcar todos los elementos de esta integridad, que ejercen su influencia en el crecimiento de la ciencia....” (p. 208.)

b) El estudio de los problemas que surgen en el proceso de desarrollo de las distintas ciencias fundamentales, así como su función en el sistema del saber, su papel y lugar en la génesis del cuadro científico del mundo, en el desarrollo de la Cultura. Entre este tipo de investigaciones se pueden destacar una serie de monografías y trabajos colectivos. Como ejemplo, sólo mencionaremos: La Astronomía y el cuadro contemporáneo del Mundo, una obra colectiva en la que se analiza cómo los nuevos descubrimientos astronómicos y las diferentes teorías que los interpretan-la Teoría del Universo Inflacionario, la Cosmología Cuántica, las Teorías de las galaxias y las Metagalaxias, el estudio de los Agujeros Negros, el desarrollo del principio antrópico o la discusión de las Hipótesis de las Civilizaciones Extraterrestres, han permitido elaborar, desde el punto de vista cosmológico, los Modelos de los posibles escenarios, que permitirán valorar las perspectivas de nuestra Civilización y la solución de los problemas globales del Mundo contemporáneo.

Algo similar, pero en un ámbito más "terrenal" se plantea en el libro La Física en el Sistema de la Cultura, un trabajo colectivo en el Prefacio del cual se señala que "La Física adquiere una importancia fundamental en el desarrollo de la concepción del mundo, el intelecto del hombre, sus capacidades y posibilidades. Es en la Física donde, en el proceso de organización de las investigaciones fundamentales, se forman las escuelas científicas de alto nivel profesional, cuya existencia sólo es posible cuando se basan en profundas normas éticas y son un reflejo de éstas» (p. 3.).

c) Una serie de profundas y bien documentadas investigaciones sobre la Historia del pensamiento cientifico (surgimiento y desarrollo de las diferentes discipli- 
nas, análisis cronológico de la evolución del pensamiento teórico, surgimiento y evolución de los complejos problemas del conocimiento científico y sus métodos, etc.) Aquí se pueden destacar: Los Tipos Históricos de Racionalidad (Vols. 1-2) en los que la racionalidad se estudia como uno de los valores fundamentales de la Cultura, un instrumento efectivo para el conocimiento de la naturaleza y la actividad social. En los dos tomos se lleva a cabo un análisis histórico del desarrollo y evolución de la racionalidad en las diferentes etapas de nuestra Cultura, desde la antigüedad clásica hasta nuestros días y se lleva a cabo una reconstrucción de los diferentes contextos históricos que la condicionan. Especial atención se presta al estudio de los periodos cruciales en la historia de la ciencia - las Revoluciones Científicas, pero también a las complejas interrelacciones entre la racionalidad y la fe religiosa, la racionalidad y la irracionalidad, la racionalidad y la libertad, etc.

Dentro de esta temática histórica son muchos los trabajos e investigaciones que estudian el desarrollo de la propia Filosofía de la Ciencia y la Tecnología y, específicamente, de estas disciplinas en Rusia. Aquí nos ha parecido interesante mencionar: La Filosofía Nacional de la Ciencia: balance previo, de E. A. Mamchur, N. F. Ovchinnikov y A. P. Ogurzov . Un libro, que según sus autores "Es un primer intento de exponer en forma sistemática la historia de la Filosofia Nacional de la Ciencia... en el que se estudian las diferentes concepciones que interpretan el desarrollo del saber cientifico en el País... prestando una atención especial a la valoración del papel de la ideología en el desarrollo de la ciencia en Rusia, asi como al análisis detallado de los diferentes criterios y sistemas metodológicos empleados para ello." (p. 2.).

Y También la Monografía de N. I. Kuznezova. Los Problemas Socio-culturales de la Formación de la Ciencia en Rusia (siglo XVIII - mediados del XIX ) En la nota editorial que acompaña la edición se exponen así las intenciones del autor: En este libro se tratan los problemas socio - culturales de la formación y el establecimiento de la ciencia rusa - desde el momento de su "arranque», con la creación de la Academia Imperial de Ciencias de San - Petersburgo hasta mediados del siglo XIX. Fundada por orden de Pedro el Grande, respondía a la urgente tarea de modernización del Pais, pero entraba en contradicción con las tradiciones establecidas en la antigua cultura rusa. Esta nueva Institución social fue creada en un medio socio-cultural desfavorable. Objetivamente la Academia fue un importante catalizador del desarrollo de la cultura rusa..." (p. 2.)

d) Sin dudas, uno de los temas de mayor actualidad entre esta abundante literatura y del que se ocupan importantes trabajos monográficos y muchos 
artículos en las revistas teóricas especializadas es el tema de la compleja relación Ciencia - Sociedad. Ahora, solo queremos mencionar algunos: El contexto socio-cultural de la ciencia, un trabajo colectivo cuyo Prefacio pretende establecer con cierta precisión el énfasis de la investigación: "La idea más importante que esta monografia quiere ofrecer al lector, consiste en que, sin menospreciar de ningún modo los méritos de la ciencia y sin poner en dudas su status epistemológicos especifico, queremos centrar su atención sobre los parámetros humanos de la actividad cientifica y exponer nuestra actitud critica hacia aquella interpretación de la ciencia, que la considera nada más que una forma específica de actividad de unos profesionales estrechos.

El pensamiento más importante de la primera parte del libro es la afirmación de que la ciencia la hacen los hombres. No la hace el sujeto transcendental, ni la Razón absoluta de Hegel, sino, precisamente el sujeto empirico, el hombre vivo "considerado desde el punto de vista de sus fines casuales" (G. Hegel), que Kant, precisamente, intenta superar. Desde este punto de vista se examinan los aspectos exotéricos, sicológicos, nacionales e individuales de la acción del cientifico y su incidencia en los resultados de la actividad cientifica" (p. 3.)

El Problema del Status Valorativo de la Ciencia a Principios del siglo XXI, es también otro trabajo colectivo que aborda, desde el punto de vista de los valores humanos, el estado actual de la ciencia y su compromiso moral con la sociedad. Como es bien conocido, desde la Epoca Moderna la ciencia ha cumplido un papel cada vez mas significativo e importante en la vida de la sociedad, desde la formación de la concepción contemporánea del mundo hasta el impulso decisivo en la realización práctica de los resultados del progreso científico-tecnológico. No obstante, en la segunda mitad del siglo XX la Ciencia y la Tecnología se han visto cuestionadas y sometidas a una severa pero inmerecida crítica y culpadas de los males de que adolece el mundo contemporáneo. El presente libro "está concebido como una respuesta a este reto crítico. En su primera parte se analizan los problemas generales del status valorativo de la ciencia: la autonomía como ideal del saber científico, el problema de la verdad, las perspectivas del desarrollo de la ciencia, el polifuncionalismo de la ciencia, la correlación entre las investigaciones fundamentales y aplicadas, etc. En la segunda parte se lleva a cabo una polémica con las tendencias y concepciones anticientíficas y seudocientificas.»(p. 2.)

e) El lugar y la función de la ciencia y la tecnologia en la sociedad contemporánea y la incidencia del progreso científico - tecnológico en la formación de la 
sociedad del futuro, es otro de los temas cuya presencia en la mayoría de las investigaciones y publicaciones se debe considerar una constante «ineludible». Un tema que, por a su actualidad, no puede ser ignorado en cualquier tipo de investigación o discurso que pretenda, de una u otra forma, valorar o interpretar el desarrollo actual del saber científico - tecnológico. Y es precisamente por esto, que este tipo de cuestiones se suelan, por regla general, plantear y discutir en forma "operativa" en infinidad de artículos, "mesas redondas", conferencias y simposios cuyos materiales al ser publicados desbordan las páginas de las revistas "tradicionales", como Voprosy Filosofii, o Cuestiones de la Historia de las Ciencias Naturales y la Técnica y han hecho necesaria la publicación desde 1999 de la Revista "Naukovedenie", que hemos traducido como Cienciología, pero que sus editores al traducirla al inglés han optado por el nombre de Sciencies Studies, definiendo su contenido así: "Naukovedenie es una revista cientifica trimestral rusa, que se publica desde 1999. Está dedicada al análisis complejo de los problemas de la ciencia nacional y mundial con los recursos de las diferentes disciplinas: Filosofia, Sociologia, Economia, Historia, Sicologia, Estadistica, Cienciometria, etc.

La finalidad de su edición es el análisis sistemático del estado y las tendencias del desarrollo de la ciencia rusa, sus tradiciones históricas y sus perspectivas, y el estudio, de gran utilidad para Rusia, de la experiencia de la organización de la ciencia en otros paises.

El carácter excepcional distintivo de esta revista es la publicación en ella de los resultados de las últimas investigaciones científicas, acompañados de su ilustración gráfica. En la revista se tratan las cuestiones del desarrollo de la comunidad cientifica, los problemas económicos de la ciencia, las cuestiones de la politica de innovaciones cientifico-técnicas, la organización de la ciencia; se analizan los problemas de la relación de la ciencia y la sociedad, la ciencia y la educación, el papel de la ciencia en la cultura, se discuten seriamente los problemas de la filosofia de la ciencia y la sociologia del saber, los aspectos informativos del desarrollo de la ciencia, la colaboración científica internacional; un interés especial representan las investigaciones sobre la historia de la ciencia y la técnica, la cienciologia. También es muy importante la orientación informativa de la revista: se publican materiales sobre la regulación normativa de la ciencia, se publican resúmenes de las conferencias cientificas, de la literatura nacional y extranjera sobre teoría de la ciencia.

La revista esta dirigida a un amplio círculo de lectores: investigadores cientificos, profesores y estudiantes de la enseñanza superior, doctorado. Los autores 
de la revista son conocidos especialistas en las diferentes ramas del saber humanistico" ${ }^{30}$.

f) La Filosofía de la Técnica y la Tecnología se ha transformado en una disciplina que no solo ha conseguido definir con cierta precisión y claridad su objeto y sus métodos, sino que se ha convertido en una asignatura, cuyo estudio resulta hoy día imprescindible en no pocas carreras del sistema de enseñanza superior. Para lo cual, durante los últimos años, en los marcos de este sistema, se han elaborado programas de estudio y diferentes manuales. En las investigaciones académicas sobre estas cuestiones se han logrado, durante estos años, serios resultados que se reflejan en un serie de monografías entre las que quisiéramos destacar: La Filosofía de la Técnica: historia y actualidad, en la que: "Se investigan las cuestiones metodológicas del saber técnico, los problemas de la técnica en el sistema de la cultura, los contextos epistemológicos y éticos de la revolución de las computadoras. Se analizan los conceptos de técnica y tecnología, la génesis de la técnica en proceso de desarrollo de la cultura. Se valoran las concepciones de la técnica en las obras de los filósofos nacionales y de otros paises. Se ofrece una concepción de la técnica como una asignatura de la enseñanza.» (p. 2.) Esta monografía se complementa con La Tecnología Tradicional y Contemporánea (análisis filosófico-metodológico), en la que se exponen los resultados de una investigación en la que use analiza la esencia de la tecnología, su diferencia e interrelación con la técnica, los factores fundamentales que condicionan la formación de la tecnología, la búsqueda de los caminos para la superación de la crisis de la civilización tecnogénica, así como algunas particularidades de la tecnología contemporánea. Los autores muestran, que el desarrollo de la tecnología está condicionado esencialmente por una serie de factores, tales como las características de la cultura, los institutos sociales, el cuadro del mundo, las formas de socialización del individuo (la educación, la vida profesional, los medios de imformación, etc..), se ofrecen ejemplos del estudio de determinadas tecnologías (de la Edad Media, la realidades virtuales, la tecnología de la información), asi como su discusión actual y las formas de su concienciación» (p. 2.).

g) Las investigaciones en el campo de la Sinergética - una nueva dirección en la evolución del conocimiento científico contemporáneo, estrechamente vinculada al desarrollo de la ciencia post-no-clásica.

$30 \quad$ Nota de la Redacción, Cienciología, N. ${ }^{\circ} 4.1999$. p. 239. 
Hoy día, no se puede pasar por alto el hecho evidente de que esta nueva disciplina, cuya definición y métodos no se ha consolidado aún definitivamente, haya encontrado su aplicación tanto no solo en una serie de ramas de la ciencia, como en importantes esferas de la práctica social. No obstante, parece evidente, que si existe cierto consenso en aceptar que el mérito de haber creado, hace más de 20 años, y "promocionado" hasta hoy día esta nueva dirección en el desarrollo del pensamiento teórico, pertenece al profesor de la Universidad de Stuttgart Herman Haken ${ }^{31}$. Cuya definición de esta nueva disciplina - la Sinergética es la teoría de la autoorganización, se puede considerar clásica, a pesar de los muchos intentos de explicitarla, complementarla o reinterpretarla.

La afirmación de Haken de que la Sinergética debería considerarse /o constituirse en una nueva metaciencia, que tendría por objeto el estudio de las leyes generales del desarrollo de todo tipo de sistemas, se basa en la idea de la existencia de cierto isomorfismo y unidad entre los diferentes modelos de sistemas particulares y este modelo «universal», lo que permite una cooperación mas amplia y estrecha entre las diferentes ciencias y sus métodos. Hay que tener presente que el surgimiento de la Sinergética no es un «invento casual», sino el resultado del desarrollo de una serie de teorías científicas cuyo papel en el siglo XX es bien conocido: desde las diferentes teorías científico-naturales "exactas" como son las teorías oscilatorias o la teoría de las ecuaciones diferenciales, hasta los métodos estructurales o sistémicos y la Teoría General de los Sistemas.

Entre las múltiples investigaciones y trabajos publicados en Rusia sobre la importancia, los problemas y el desarrollo de esta nueva disciplina destacaremos, como conclusión a esta enumeración de temas y problemas que se plantean actualmente ante la filosofía de la ciencia y la tecnología en este País, la monografía de V. I. Arshinov La Sinergética como un fenómeno de la Ciencia Post-no-clásica, y la monografía colectiva La Ontología y la Epistemología de la Sinergética, en cuya Introducción se dice: "La intención de los autores ha con-

31 En Rusia los libros de H. Haken se conocen muy bién y están traducidos al ruso desde los años 80. Sinergética, fue editada en Moscú por la Editorial MIR en 1980; La Sinergética: jeraquia de la inestabilidad en los sistemas de autoorganización, por la misma Editorial en 1985; Información y autoorganización: El rnfoque macroscópico de los sistemas complejos,por la misma y en el mismo lugar en 1991. Entre sus útimos trabajos aún no editados en ruso, queremos mencionar: HAKEN H. Principles of Brain Functioning. A sinergetic Aproach to brain activity behavior ahd cognition. Sprimger, 1996. 
sistido en demostrar, que la Sinergética al ser un modelo de autoorganización, aporta nuevas posibilidades, estrategias y estilos de pensamiento, que ofrecen enfoques no tradicionales a muchos de los problemas y modos de pensar del filosofar clásico. Los autores mantienen la esperanza, de que el arsenal conceptual-imaginativo de la Sinergética pueda ser incorporado a la formación del núcleo de los fundamentos de los enfoques post-no-clásicos de las estrategias del filosofar y ser una fuente de innovaciones en la metodología de la creatividad cientifica.» (pp. 3 - 4). 